\title{
The elemental hues of short-wave and extraspectral lights
}

\author{
KENNETH FULD \\ University of New Hampshire, Durham, New Hampshire 03824 \\ and \\ B. R. WOOTEN and JAMES J. WHALEN \\ Brown University, Providence, Rhode Island 02912
}

\begin{abstract}
The continuous judgmental color-naming technique was used to assess the elemental nature of hue names descriptive of short-wave and extraspectral lights. Subjects were instructed to describe the hue of a 3-deg, 1-sec, 1,000-Td light by assigning percentages to each of three or four response categories available for use in a particular session. Response categories were chosen from the following group: red, green, blue, yellow, violet, and purple. Test stimuli consisted of monochromatic lights ranging from 510 to $420 \mathrm{~nm}$ and various proportions of 400and 700-nm light. Results from eight subjects showed the color names blue, red, and green to be necessary and sufficient in describing these lights. On the basis of criteria established for the elementalness of hues, blue, red, and green were determined to be elemental, whereas purple and violet were found not to be elemental.
\end{abstract}

Modern accounts of color perception based on an opponent process model in conjunction with an initial trichromatic stage (Guth, Massoff, \& Benzschawel, 1980; Hurvich \& Jameson, 1957; Walraven, 1962) assert that there are four psychologically elemental hues ${ }^{1}$ perceived throughout the spectrum: blue, green, yellow, and red. The precedent for this idea dates back to the 19th century (Aubert, 1865; Donders, 1881; Hering, 1874; Mach, 1865; Müller, 1896-all cited in Boring, 1942). Surprisingly, this fundamental postulate has never been fully tested. In fact, it has not been entirely accepted. Some investigators (Beare, 1963; Beare \& Siegel, 1967; Thomson, 1954) have either implicitly or explicitly argued for the inclusion of orange and/or violet or purple in the domain of elemental hues.

That orange does not belong in this category was convincingly demonstrated by Sternheim and Boynton (1966). In their study, subjects used a continuous judgmental color-naming technique that yielded a description of mid- and long-wave monochromatic lights in terms of the proportionate values of their constituent hues. For a given session, a subject would be asked to choose from an available set of hueresponse categories and to describe individual lights (ranging from 530 to $620 \mathrm{~nm}$ ) by assigning values to these categories that reflected the proportion of green, yellow, orange, or red perceived in each light. Sessions differed in terms of which hue-response categories (green, yellow, orange, red) were available for the subject to use. Thus, for each light they investigated, they were able to obtain a description of its perceptual components, limited only by the set of hue names from which the subjects were allowed to choose. As an example, when the categories green, orange, and red were available for use, a subject might have described the perceptual content of a light of $600 \mathrm{~nm}$ as consisting of $76 \%$ orange and $3 \%$ red. The same light might have been described by the subject as consisting of $77 \%$ red and $23 \%$ yellow, however, if he were allowed the hue names green, yellow, and red. Note that, in the former case, the percentages did not sum to 100 . The degree to which the ratings fell short of $100 \%$ varied with wavelength and allowed hue-naming categories.

A computed function could be derived by subtracting the sum of the ratings for each light from 100. One of the criteria Sternheim and Boynton established for determining whether or not a hue was considered to be elemental was that such a computed function be obtainable when that particular hue name was prohibited in a given session, and that this computed function be nearly identical to the response function obtained when this hue name was allowed. Another criterion employed to determine the elementalness of a hue was that, in the resulting naming functions, the maximum response of a hue category was to appear in a region of the spectrum in which neighboring categories were at a minimum.

On the basis of the above criteria, Sternheim and Boynton concluded that the hue name orange did not represent an elemental hue. They established that the mid- and long-wave end of the spectrum could be described adequately by the hue names green, yellow, and red, and that the hue name orange was not required. 
The question remains concerning the extent to which purple or violet can be considered elemental with regard to hues seen in the short-wave end of the spectrum and extraspectral lights. The aim of the present study was to answer this question by applying the continuous judgmental technique as used in the Sternheim and Boynton experiment. The demonstration that subjects can adequately describe these lights with the hue names red and blue, and that the hue names purple and violet are not necessary, coupled with Sternheim and Boynton's findings, would substantiate the major postulate of all opponent process models of color vision that address the question of hue-that there are four and only four elemental hue sensations: blue, green, yellow, and red.

\section{METHOD}

\section{Subjects}

Eight experimentally naive subjects were used. Their color vision was assessed as normal on the basis of results from the administration of the HRR Pseudoisochromatic Test Plates and the Farnsworth Dichotomous Panel D-15 Test.

\begin{abstract}
Apparatus
A standard, two-channel Maxwellian view optical system was used. Light was provided by a 1,000-W xenon-arc lamp and was rendered monochromatic in each channel by a Bausch \& Lomb grating monochromator (nominal half-bandwidth $=6.6 \mathrm{~nm}$ ). Blocking filters were used below $500 \mathrm{~nm}$. When extraspectral stimuli were used, special care was taken to superpose the shortand long-wave components by precisely positioning an achromatizing lens located at a place in the optical system conjugate with the pupil of the subject's eye. The alignment of the subject's eye with respect to the focal point of the light source was achieved and maintained by use of an adjustable bite-bar and headrest assembly.
\end{abstract}

\section{Procedure}

The continuous judgmental technique was used to determine the elementalness of perceived hues. The subjects viewed a series of test flashes of different spectral composition that had been approximately equated for brightness. Each subject gave percentages to the allowed hue names comprising his or her perception of these lights. There were two major conditions. In the first condition (five subjects: three males, two females), the use of the purple hue-naming category was investigated. The violet huenaming category was investigated in the second condition (three subjects: one male, two females). Different hue-naming categories were allowed in each session. For the purple condition, they were as follows: Day 1-red, green, and yellow; Day 2-red, blue, and green; Day 3-red, purple, and green; Day 4-red, purple, blue, and green. For the violet condition, the subjects were allowed the following categories: Day 1-blue, green, and yellow; Day 2-red, blue, and green; Day 3-red, violet, blue, and green. Each session lasted about $1.5 \mathrm{~h}$. In addition, the subjects participated in a 30 to 45 -min practice session. They were shown between 10 and 30 different lights, chosen randomly, and were asked to assign percentages to the allowed hue categories, which had been arbitrarily chosen for this occasion.

An experimental session consisted of a 10-min dark-adaptation period followed by eight presentations, selected randomly, of each of 16 different test stimuli. Table 1 gives the spectral composition of these test lights. Each light, a 3-deg circular field, was presented for $1 \mathrm{sec}$ to the fovea of the subject's right eye. Stimuli were approximately equated for brightness on the basis of Wagner and Boynton's (1972) relative spectral efficiency function determined by the direct comparison method. The brightness was equal to that of a $1,000-\mathrm{Td}, 580-\mathrm{nm}$ light (measured according to the method of Westheimer, 1966). Stimuli of different spectral composition were presented successively, with a minimum time interval of $15 \mathrm{sec}$. If, for some reason, however, the subject wished to see the same light again, a signal would result in the presentation of the same stimulus $10 \mathrm{sec}$ later. This occurred very infrequently. The subjects were told that, when viewing each stimulus, they should treat the total amount of hue produced by the light as having a value of 100. In describing the perceptual content of the light, they were asked to assign percentages to each of the hue categories available for use in a particular session. They were told to report a value for each category, even if it was zero. It was emphasized that their ratings need not necessarily add up to 100 . In addition, the subjects were instructed to ignore saturatıon.

\section{RESULTS AND DISCUSSION}

Results from the condition in which the hue name purple was examined are presented in Figures 1 through 6. The mean hue-naming response of five subjects (eight responses per subject) is shown for each of the 16 different test lights, which are represented along the axis of abscissas in Farbton (DIN color system). ${ }^{2}$ The wavelength or complementary wavelength of these lights, as well as their CIE chromaticity coordinates, are given in Table 1 .

Figure 1 presents the results from the session in which the response categories red, green, and yellow were allowed. The absence of a yellow response function indicates that subjects saw no yellow in any of these lights. Red and green response functions do appear, however, as well as a computed function, which is obtained from the difference between the summed response for a given light and the value 100 . The red, green, and computed function all peak at a point at which other functions are at their minimum.

Figure 2 shows the results for the session in which the hue names red, blue, and green were available for

Table 1

Specification of Test Lights

\begin{tabular}{ccccc}
\hline & & \multicolumn{2}{l}{$\begin{array}{l}\text { CIE Chromaticity } \\
\text { Coordinate }\end{array}$} & \\
\cline { 3 - 4 } length & $\begin{array}{c}\text { Complementary } \\
\text { Wavelength }\end{array}$ & $\mathrm{x}$ & $\mathrm{y}$ & Farbton \\
\hline 510 & & .014 & .750 & 21.4 \\
500 & & .008 & .538 & 20.8 \\
490 & & .045 & .295 & 19.5 \\
480 & & .091 & .133 & 17.7 \\
460 & & .144 & .030 & 14.6 \\
440 & & .164 & .011 & 13.9 \\
420 & & .171 & .005 & 13.7 \\
400 & 567 & .173 & .005 & 13.5 \\
& $566^{*}$ & .192 & .014 & 13.3 \\
& $565^{*}$ & .206 & .020 & 13.0 \\
& $558^{*}$ & .269 & .049 & 12.2 \\
& $543^{*}$ & .342 & .083 & 11.2 \\
& $501^{*}$ & .502 & .156 & 10.1 \\
& $497^{*}$ & .572 & .189 & 9.7 \\
700 & $494^{*}$ & .676 & .237 & 9.0 \\
& 492 & .735 & .265 & 8.5 \\
\hline
\end{tabular}

*Various proportions of $400+700$. 


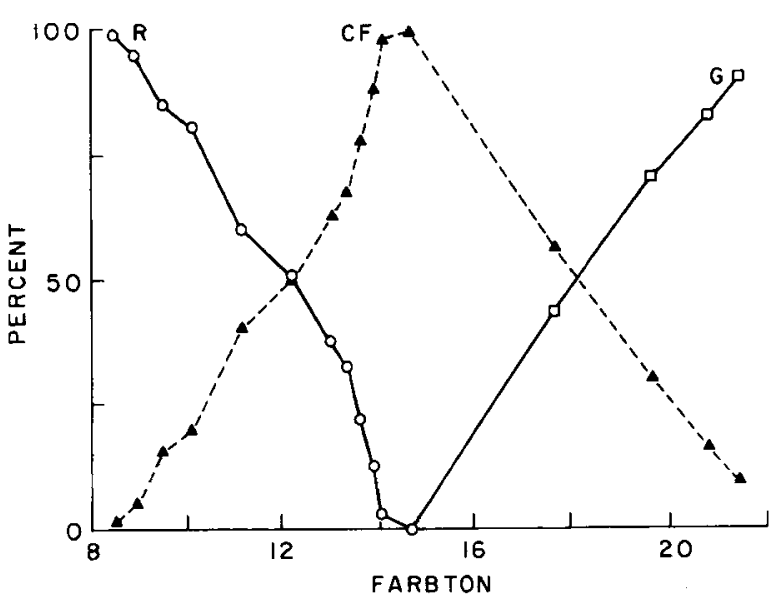

Figure 1. Hue-naming responses for the purple condition, Day 1 (allowed hue names: red, green, yellow). Red and green response functions are indicated by circles and squares, respectively. Filled triangles (dashed curve) represent the computed function.

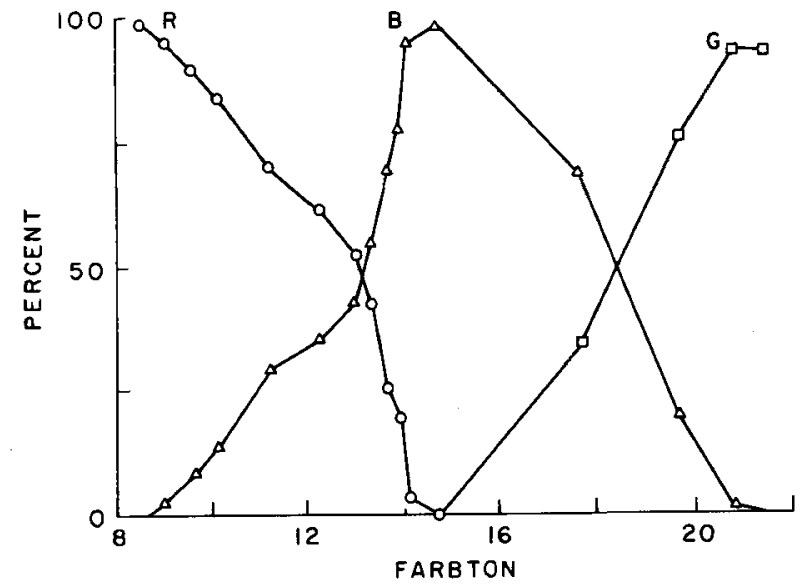

Figure 2. Hue-naming responses for the purple condition, Day 2 (allowed hue names: red, blue, green). Red, blue, and green response functions are indicated by circles, triangles, and squares, respectively.

use by the subject. Again, each function reaches a maximum at a point at which other functions are at their minimum. No significant computed function is obtainable, since the sum of the responses for each light is $100 \%$, or nearly so. Thus, the hue name purple fails to fulfill one of the criteria for an elemental hue: that a computed function be obtainable when that particular response category is prohibited from use.

In contrast with the above, when the hue name blue was prohibited from use in Day 1, a computed function was obtainable (Figure 1) and closely matches the blue response function in Figure 2. This comparison is illustrated in Figure 3 and supports the elemental nature of the hue name blue.

Figure 4 shows the results for the session in which the hue names red, purple, and green were allowed.
Although each function peaks at a point at which other functions are at their minimum, subjects, on the average, are unwilling to call any light $100 \%$ purple. A function can be computed from these data that closely resembles the blue function obtained when the hue names red, purple, blue, and green were allowed. Results from this session are shown in Figure 5.

It can be seen from Figure 5 that lights formerly described as red and blue (Figures 1 and 2) are now described as purple and red or purple and blue. Whereas the green response function remains unaffected by the availability of the categories blue and purple, the red and blue response functions are affected. The addition of the purple hue-naming category reduces the proportion of red and blue responses to lights that are now described as purple

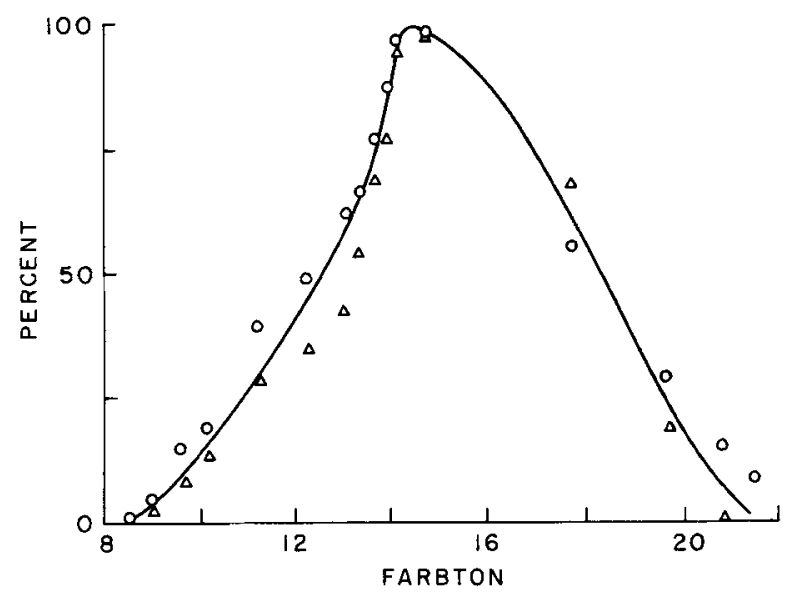

Figure 3. Comparison between the computed function from Day 1 (circles) and the blue response function from Day 2 (triangles) of the purple condition. Curve is fit by eye.

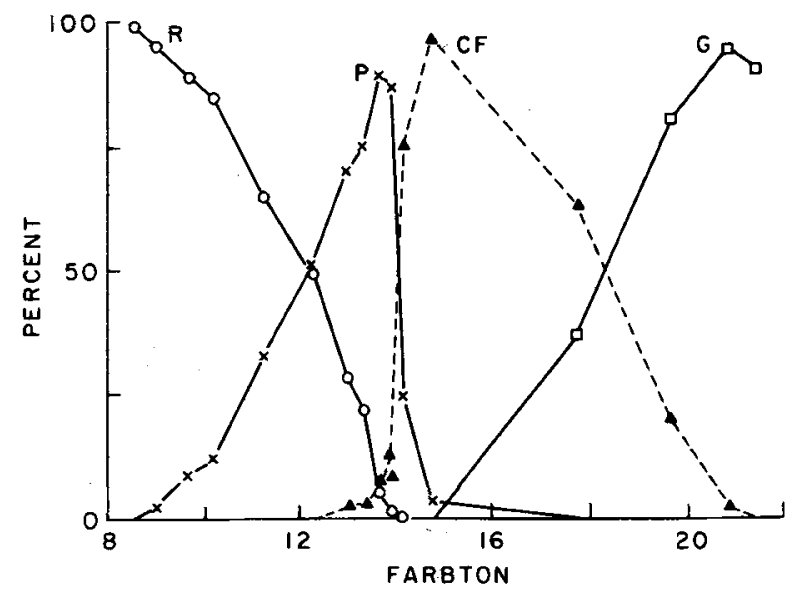

Figure 4. Hue-naming responses for the purple condition, Day 3 (allowed hue names: red, purple, green). Red, purple, and green response functions are indicated by circles, crosses, and squares, respectively. Filled triangles (dashed curve) represent the computed function. 


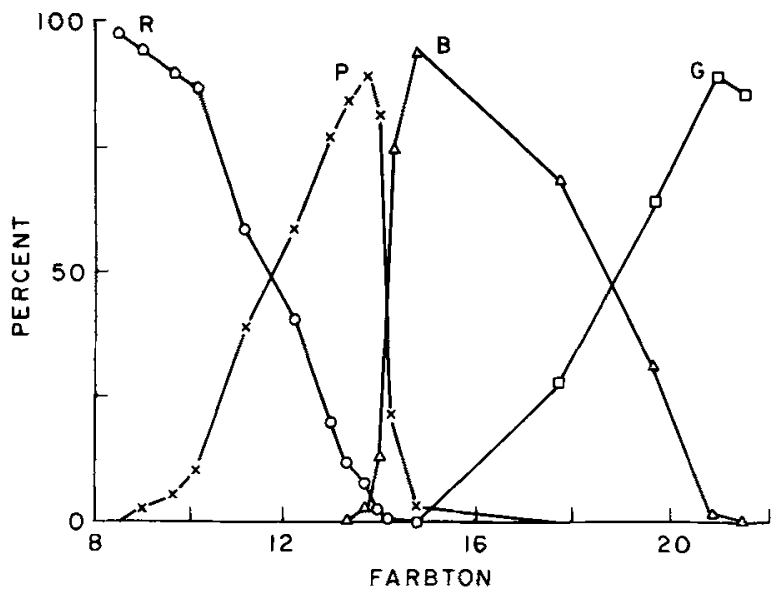

Figure 5. Hue-naming responses for the purple condition, Day 4 (allowed hue names: red, purple, blue, green). Red, purple, blue, and green response functions are indicated by circles, crosses, triangles, and squares, respectively. (o)

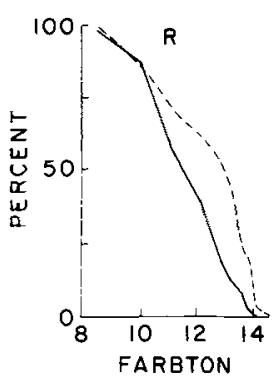

(b)

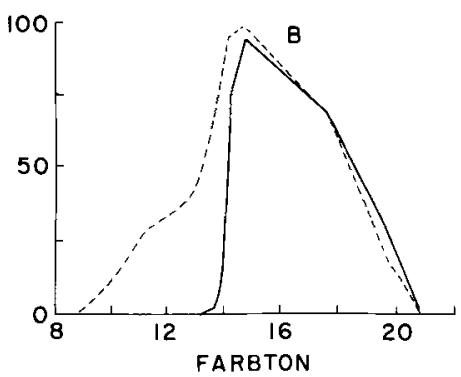

Figure 6. Comparison of red response functions (a) and blue response functions (b) from sessions in which purple was allowed (solid curves) and was not allowed (dashed curves).

to some degree. This is more evident in Figure 6, which compares the red and blue response functions from sessions in which purple was allowed (solid curve) and was not allowed (dashed curve). The addition of the purple response category has the effect of steepening the slopes of the red and blue response functions at points at which the two functions would otherwise overlap.

Results from the second major condition, in which the hue name violet was investigated, are shown in Figures 7 through 9. Data for the session in which the hue names blue, green, and yellow were available for use by the subject are presented in Figure 7. Red and violet were not allowed. As on Day 1 of the purple condition (Figure 1), the subjects here do not perceive yellow in any of these lights. Functions peak at a point at which others are at their minimum, including the computed function, which closely resembles the red response function in Figure 8.

Figure 8 presents the results from the session in which the categories red, blue, and green were al- lowed. With the use of the violet category prohibited, no appreciable computed function is obtainable. This illustrates again that these lights can be fully described by the hue names red, blue, and green.

Figure 9 presents a comparison of the red response function from Figure 8 with the computed function from Figure 7. The close correspondence between the two curves suggests the elemental nature of red.

For the session in which the categories red, violet, blue, and green were allowed (Figure 10), there was less of a tendency for subjects to use the hue names red and blue. That is, the slopes of the red and blue response functions are steeper when the category of violet is also available for use (Figure 10) than when violet is not allowed (Figure 8). This is analogous to the findings from the purple condition.

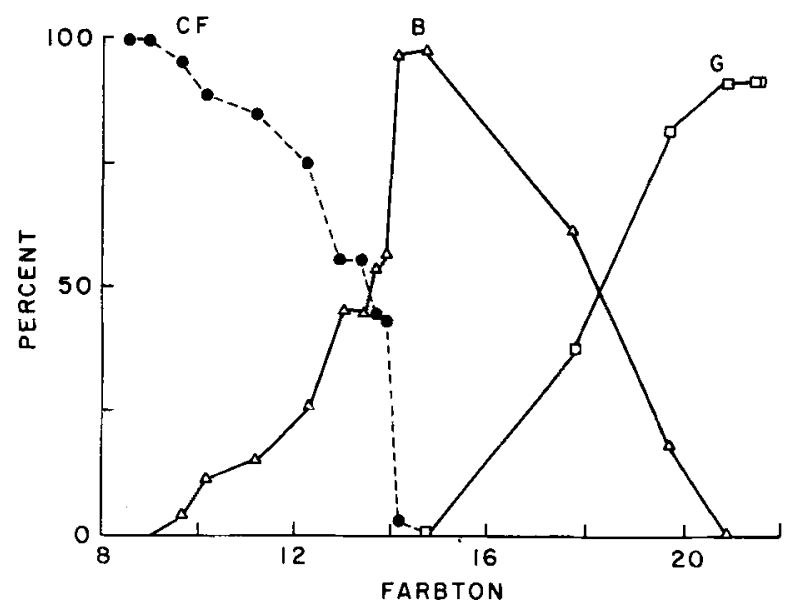

Figure 7. Hue-naming responses for the violet condition, Day 1 (allowed hue names: blue, green, yellow). Blue and green response functions are indicated by triangles and squares, respectively. Filled circles (dashed curve) represent the computed function.

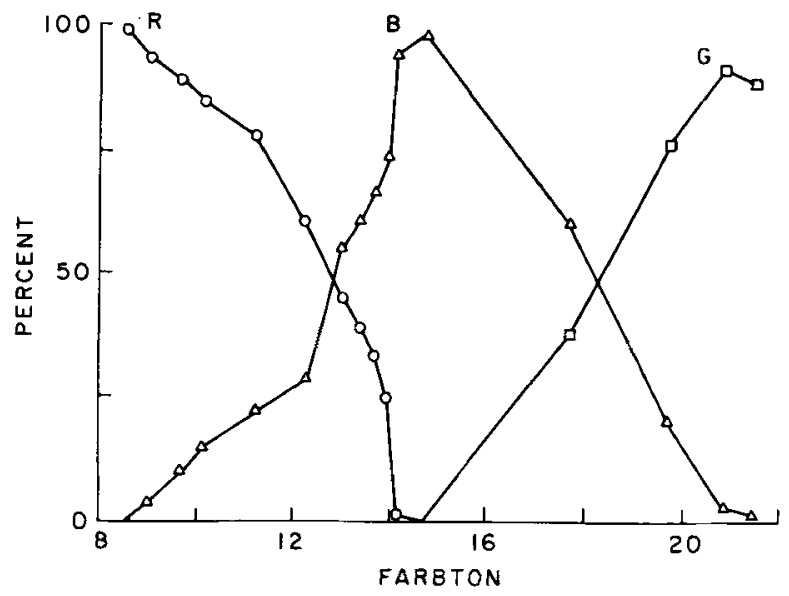

Figure 8. Hue-naming responses for the violet condition, Day 2 (allowed hue names: red, blue, green). Red, blue, and green response functions are indicated by circles, triangles, and squares, respectively. 


\section{GENERAL DISCUSSION}

On the basis of the criteria established for the elementalness of hues, it is concluded that purple and violet do not represent elemental hues in the same sense as do red, blue, and green. First, in every case, red, blue, and green response functions peak at a point at which other response functions are at their minimum. This is not always true for the purple and violet response functions. Second, functions can be computed, when the use of red and blue hue-naming categories is prohibited, that closely resemble the actual red and blue response functions. This is not

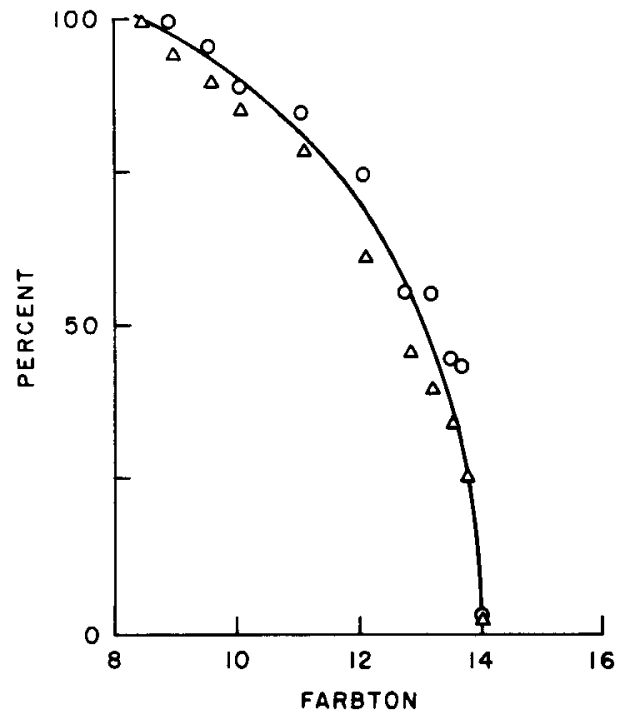

Figure 9. Comparison between the computed function from Day 1 (circles) and the red response function from Day 2 (triangles) of the violet condition. Curve is fit by eye.

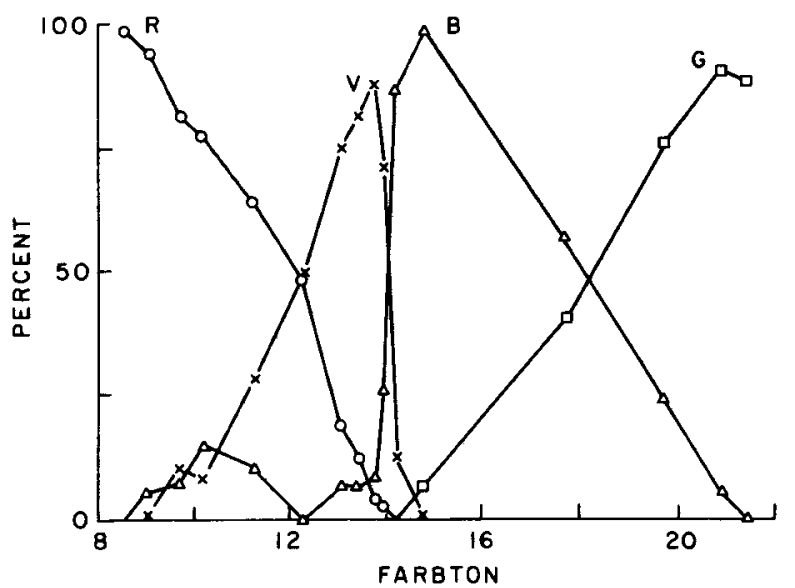

Figure 10. Hue-naming responses for the violet condition, Day 3 (allowed hue names: red, violet, blue, green). Red, violet, blue, and green response functions are indicated by circles, crosses, triangles, and squares, respectively.

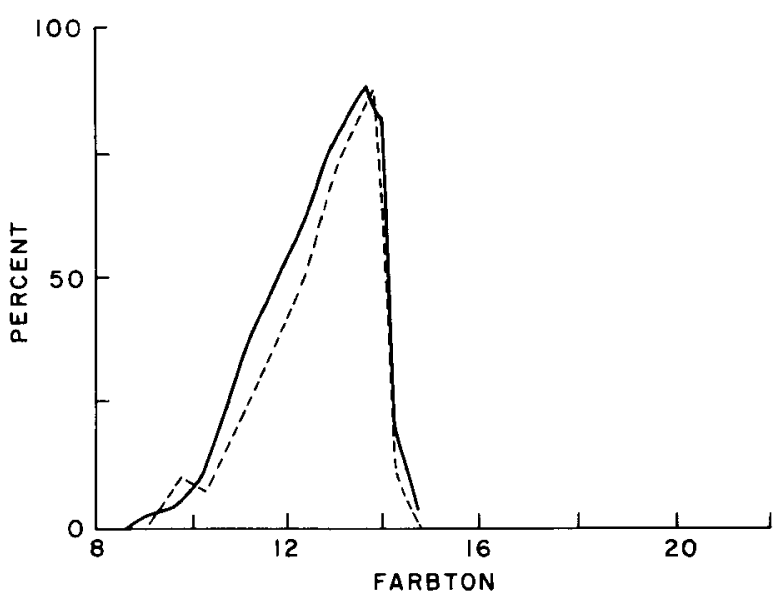

Figure 11. Comparison between purple response function (Day 4, purple condition) and violet response function (Day 3, violet condition).

at all true for purple and violet. Thus, for describing short-wave and extraspectral lights, the hue names red, blue, and green are necessary and sufficient. Whereas purple and violet can be descriptive of some of these lights, these hue names are not required and are, in fact, redundant with the compound name red/blue.

Purple and violet are also redundant with respect to one another. That these two names are used interchangeably can be seen in Figure 11, which compares the purple and violet response functions from two different groups of subjects. This is in agreement with Chapanis (1965), whose subjects made virtually no distinction between these two colors.

The present results show that when only elemental hue names are available for use by subjects, the slopes of the resulting response functions are all roughly equal to one another (excluding sign) and that this slope is approximately equal to 2 (Figures 2 and 8 ). This is not true when non-elemental hue names are allowed, as can be seen in Figure 4. Here, the negative slope of the purple function is clearly steeper than that of the red or green response functions. Thus, another criterion for the elementalness of a hue might be that when the continuous judgmental color-naming technique is used and the resulting functions are plotted in terms of Farbton, the slopes of these functions should be approximately equal to 2. We find this, in general, to be true for yellow, green, and red, but not for orange, when Sternheim and Boynton's data are plotted in this fashion.

\section{REFERENCES}

Beare, A. C. Color-name as a function of wavelength. American Journal of Psychology, 1963, 76, 248-256.

Beare, A. C., \& Siegel, M. H. Color name as a function of 
wavelength and instruction. Perception \& Psychophysics, 1967, 2, 521-527.

Boring, E. G. Sensation and perception in the history of experimental psychology. New York: Appleton-Century-Crofts, 1942.

Chapanis, A. Color names for color space. American Scientist, $1965,53,327-346$.

Guth, S. L., Massoff, R. W., \& Benzschawel, T. Vector model for normal and dichromatic color vision. Journal of the Optical Society of America, 1980, 70, 197-212.

HuRvich, L. M., \& JAMESON, D. An opponent-process theory of color vision. Psychological Review, 1957, 64, 384-404.

Sternheim, C. E., \& Boynton, R. M. Uniqueness of perceived hues investigated with a continuous judgmental technique. Journal of Experimental Psychology, 1966, 72, 770-776.

Thomson, L. C. Sensations aroused by monochromatic stimuli and their prediction. Optica Acta, 1954, 1, 93-101.

W AGNER, G., \& Boynton, R. M. Comparison of four methods of heterochromatic photometry. Journal of the Optical Society of America, 1972, 62, 1508-1515.

WalRaven, P. L. On the mechanisms of colour vision. Soesterberg: Institute for Perception RVO-TNO, 1962.
Westhe imer, G. The Maxwellian view. Vision Research, 1966, 6, 669-682.

\section{NOTES}

1. In using the term "elemental hue," we are referring to a sensation that is irreducible. For example, it has been argued that the sensation orange is a compound hue consisting of the elemental hues yellow and red. A light that elicits the sensation of yellow, however, can be described in no other terms. In such a case, in which only a single elemental hue results, the hue is unique.

2. Plotting the data in the more conventional way (in terms of wavelength and complementary wavelength) would have resulted in a particularly uneven spacing of the extraspectral stimuli along the axis of abscissas. For this reason, we chose to express our lights in terms of Farbton, the units of which divide the hue circle into 24 perceptually equal intervals.

(Received for publication November 4, 1980; accepted February 10, 1981.) 